\title{
Intervention patterns of pivot nurses in oncology
}

by Myriam Skrutkowski, Andréanne Saucier, Judith A. Ritchie, Ngoc Tran and Kevin Smith

\begin{abstract}
The Pivot Nurse in Oncology (PNO) is a health care professional dedicated to providing patients with cancer and their families with continuing and consistent supportive care throughout the care trajectory. The purpose of this paper is to describe the variation and frequency of nursing interventions delivered by 12 PNOs at our health centre. An administrative analysis over a three-year period revealed a total of 43,906 interventions that were grouped into 10 categories. This analysis provided a description of the intervention frequency and these interventions were further collapsed into the four role functions of the PNO. Coordination/continuity of care and the assessment of needs and symptoms were identified as the dominant practice domains of the PNO in the professional cancer navigator role.
\end{abstract}

\section{Introduction}

The Quebec Ministry of Health and Social Services (MSSS) implemented the role of the Pivot Nurse in Oncology (PNO) in 2005 across the province. The role, referred to in Quebec as the IPO (infirmière pivot en oncologie), has been created in response to the needs identified by patients affected by cancer. Those needs include accessibility to a resource person specialized in cancer care who is able to facilitate access to different services, respond to queries, and provide information and support at any point throughout the patient's cancer journey. As a member of the interdisciplinary team, the PNO has four practice functions-

\section{About the authors}

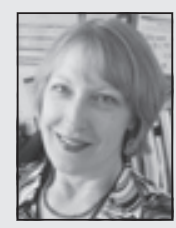

Myriam Skrutkowski, RN, MSc, CON(C), Clinical Nurse Specialist Oncology, McGill University Health Centre (MUHC), 1650 Cedar Avenue, Room T6.212, Montreal, QC H3G 1 A4

tel: 514-934-1934, ext. 43085; fax: 514-934-8408; email: myriam.skrutkowski@muhc.mcgill.ca

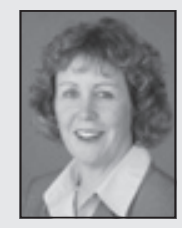

Andréanne Saucier, RN, MSc, CON(C), Associate Director of Nursing for the Cancer Care Mission and Respiratory Care in the Department of Nursing, MUHC, Montreal, QC

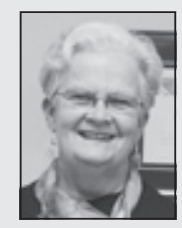

Judith A. Ritchie, RN, PhD, Associate Director for Nursing Research in the Department of Nursing, MUHC, Montreal, QC

Ngoc Tran, BSc, Information System Analyst for the Cancer Care Mission in the Department of Nursing, MUHC, Montreal, QC

Kevin Smith, RN, BN, MSc(A), Clinician Nurse, ICU, Royal Victoria Hospital, MUHC, Montreal, QC assessment of needs and symptoms, teaching, support, and coordination/continuity of care. Recent articles have described the vision of practice and implementation of the PNO, yet there is scarce examination of nursing interventions. Information about the intervention variation and frequency can provide insight on the patterns of nursing care that the PNO provides. The purpose of this article is to describe the actual interventions delivered to patients with cancer by the PNOs at a tertiary care university health centre in Quebec.

\section{Literature review}

The diagnosis of cancer is a life-altering situation for an individual. Patients with cancer and their families face enormous challenges related to learning about the disease and making complex decisions about treatment, all the while finding ways to cope with the emotional and financial realities encountered with cancer (Ferrante, Chen, \& Kim, 2008; Plante \& Joannette, 2009; Saegrov \& Halding, 2004). Health care professionals such as nurses can provide supportive care to address patient needs, described as including the physical, social, emotional, informational, psychological, spiritual, and practical needs that occur over the phases of the cancer journey (Fitch, Porter, \& Page, 2009). As the needs vary from one person to another and change over the course of the cancer journey, some of the major challenges patients face may be due to a lack of continuity of care (Dumont, Dumont, \& Turgeon, 2005). Continuity of care has been previously described as the delivery of patient and family care in a manner that is seamless, "coherent, connected, and consistent with the patient's medical needs and personal context" (Haggerty et al., 2003; Lohfeld, Brazil, \& Willison, 2007).

One solution discussed in the literature to address the lack of continuity of care is the utilization of patient navigators. The patient navigator role first originated in Harlem, New York, where Dr. Freeman identified the unmet needs of African-American women who were trying to access timely care. That patient navigator was a layperson whose role was to assist patients with accessing services in a timely and organized fashion (Freeman, Muth, \& Kerner, 1995). Subsequent authors have provided a variety of descriptions of this role, along with an array of possible educational and professional requirements needed to enact the role (Schwaderer \& Itano, 2007; Rahm, Sukhanova, Ellis, \& Mouchawar, 2007; Fischer, Sauaia, \& Kutner, 2007).

The Canadian Partnership against Cancer (CPAC) advocates that the navigator can be either a health professional from any discipline or a layperson, as determined by the program objectives (Canadian Partnership against Cancer, 2010). In Quebec, the navigator is a nurse with oncology expertise, who is involved from the diagnosis and onwards throughout the trajectory of care (Fillion et al., 2009; Fillion et al., 2006; Plante \& Joannette, 2009). The nurse not only incorporates the functions of a patient navigator (i.e., coordination of care, overcoming accessibility issues), he/she also provides the patients and their families with bio-psychosocial assessment, support, and education/teaching (de Serres \& Beauchesne, 2000; Plante \& Joannette, 2009). While there are descriptions of what functions the navigator should undertake, there is no empirical evidence of what is actually done in practice (MSSS, 2008; Fillion et al., 2009; CPAC, 2010). Therefore, we did this administrative analysis to describe the actual practice of the PNO. 
Cancer Control Quebec, referred to as the Direction de la lutte contre le cancer (DLCC), formally implemented the PNO role and provided some dedicated funds to create this new position. The PNO accompanies patients and families from diagnosis of cancer onward. They work with a particular population being followed by the employing hospital, regardless of phase of the trajectory, settings, or treatment being received. The PNO has four practice functions: a) needs/symptoms assessment and follow up, b) information delivery/teaching, c) providing support, and d) coordination of care and services (MSSS, 2008). The decision to implement this as a nursing role was based on the recommendation of the provincial oncology nursing consultation group (Ministère de la Santé et des Services sociaux (MSSS), 2005) that the PNO has a baccalaureate degree and a background of clinical oncology nursing expertise including bio-psychosocial knowledge of oncology. In order to perform this role, an individual is expected to have the following qualifications: a) clinical competencies in oncology, b) education in bio-psychosocial assessment, c) knowledge of family dynamics, d) skills in therapeutic relationships and team work, and e) leadership skills (de Serres \& Beauchesne, 2000).

In 2005, eight PNO positions were created at our health centre and each PNO was assigned to a specific population with cancer (i.e., breast, prostate, lung, colorectal, gynecology, head and neck, hematology, and young adults). There are descriptions of the required processes and experiences involved with the implementation of the PNO into an interprofessional team environment (Plante \& Joannette, 2009; Fillion et al., 2006). Recently, others (Fillion et al., 2009; CPAC, 2010) have described the role of the PNO by using a bi-dimensional Professional Navigation Framework. This framework describes the role as involving two primary dimensions: (a) organizational activities that involve facilitating continuity of care, and (b) clinical activities that involve promoting patient and family empowerment.
As described above, the PNO provides a variety of interventions to patients and their families. However, there is a paucity of research that has focused specifically on the PNO role, the interventions they provide, and the patient outcomes. Previous research has identified the effectiveness of interventions in addressing such issues as caregiver strain and burden (Honea et al., 2008), cancer fatigue (Mitchell, Beck, Hood, Moore, \& Tanner, 2007), treatment adherence (Miaskowski, Shockney, \& Chlebowski, 2008), emotional distress (Allard, 2007), and the patient-partner relationship (Morgan, 2009). However, we found no reports about whether practitioners, including the PNO, actually enact such interventions.

There has been some research that examined a PNO's impact on selected outcomes with patients with breast and lung cancer (Skrutkowski et al., 2008), and patients with head and neck cancer (Fillion et al., 2009). In a study about professional patient navigation for those with head and neck cancer, the results indicate an association between the presence of PNO and continuity of care (higher satisfaction and shorter hospitalization) and empowerment (fewer related problems and better emotional quality of life) (Fillion, 2009). However, there has been no description of the enacted activities and interventions by the PNO. Such a description could provide a better basis for developing studies to determine the connection between the interventions the PNOs provide and their impact on patient outcomes. This paper describes the patterns of PNO practice including the frequency and types of interventions, as revealed through an administrative audit of nursing documentation.

\section{Method}

We conducted an audit of an administrative database using Medivisit ${ }^{\circledR}$, an existing hospital booking system that permitted the tracking of ambulatory clinical activities for administrative purposes. Each PNO entered data reflecting interventions for each patient encounter using the intervention documentation

\begin{tabular}{|c|c|}
\hline Intervention & Description \\
\hline Administration or clerical & $\begin{array}{l}\text { Administrative work or clerical; appointments; faxing prescriptions or documents; verifying } \\
\text { that tests are booked. }\end{array}$ \\
\hline Advocacy for the patient & $\begin{array}{l}\text { Speaking on behalf of the patient; mobilizing the physician; influence other health care } \\
\text { professionals; change or modify the care plan, e.g., community clinic, emergency room. }\end{array}$ \\
\hline Continuity of care & $\begin{array}{l}\text { Coordination of care with services and professionals; communication on treatment plan and } \\
\text { follow-up of patients; plan of care discussed at interdisciplinary rounds; reporting back to } \\
\text { team. }\end{array}$ \\
\hline Data gathering about new patient & $\begin{array}{l}\text { Initial patient assessment for baseline profile on past medical history, cancer diagnosis, coping } \\
\text { skills, available support and resources. }\end{array}$ \\
\hline Family assessment & Genogram present; social support assessment. \\
\hline Monitor/surveillance & Follow-up of changes with symptoms assessed initially; review of symptom outcomes. \\
\hline Referral/consult & $\begin{array}{l}\text { Formal request to service or health care professional (community, emergency room, palliative } \\
\text { care, family doctor...). }\end{array}$ \\
\hline Support to family and patient & $\begin{array}{l}\text { Review of coping skills; giving information in order to help make decisions; support to express } \\
\text { emotion; giving encouragement. }\end{array}$ \\
\hline $\begin{array}{l}\text { Symptom assessment- } \\
\text { new symptoms only }\end{array}$ & $\begin{array}{l}\text { Assessment of symptoms identified or reported; use of symptom grading scale (0 to 10) by } \\
\text { patient. }\end{array}$ \\
\hline $\begin{array}{l}\text { Teaching (as a process) } \\
\text { management of symptoms }\end{array}$ & $\begin{array}{l}\text { Strategies to improve symptoms, e.g., energy saving for fatigue, e. g., anti-emetic medication } \\
\text { for nausea. }\end{array}$ \\
\hline
\end{tabular}


tool described below. Information was stored using the hospital's computerized data system on a continuous basis for a three-year period.

The intervention documentation tool was developed following a chart review of the practice functions of a PNO during a research study (Skrutkowski et al., 2008). The PNO's practice could be described, using a content analysis of the PNO's documentation, in nine categories of nursing interventions that were adapted from the intervention categories of the Omaha System. This research-based Omaha System comprises a standardized taxonomy that is used to classify and document various facets of nursing care that may be provided to patients (Martin, 2004). Our documentation tool consisted of nine nursing intervention categories and one category, administrative/clerical, to track the extent of non-nursing tasks (Refer to Table 1). The descriptions for each intervention were based on the activities cited in the PNOs' documentation.

Table 2. Organization of 10-category

list into the PNO role functions

Nursing Interventions Checklist
adapted from the Omaha System

(Martin, 2004)

Data gathering

Family assessment

Monitor surveillance

Symptom assessment

\begin{tabular}{|l|l|}
\hline Support to family \& patient & 2. Support \\
\hline Teaching management of symptoms & 3. Teaching/information \\
\hline $\begin{array}{l}\text { Administration or clerical } \\
\text { Advocacy } \\
\text { Continuity of care }\end{array}$ & $\begin{array}{l}\text { 4. Continuity/ } \\
\text { coordination }\end{array}$ \\
\hline
\end{tabular}

\section{Documentation processes}

One year after the introduction of the PNO role at the MUHC, the PNOs were required to document their interventions based on the 10 categories of nursing interventions described above for administrative and quality monitoring processes. The Clinical Nurse Specialist (CNS) in Oncology provided training to the PNOs about the intervention schema during the PNOs' orientation period. The CNS continued to provide information and support to each PNO regularly with either one-to-one sessions or discussions in monthly group meetings. These discussions ensured consistency with the use of the intervention categories and fostered the PNOs' understanding about how to use the tool to describe their interventions. The PNOs indicated that they were able to capture all their interventions with the tool. Immediately after the completion of a phone call or in-person encounter with a patient and/or family member, the PNO documented a summative record of the interventions completed. They were instructed to follow the written description of each category to guide them during the documentation process. In addition, for every intervention documented in the chart, they entered the appropriate intervention category into the electronic database. From 2006 to 2009, each PNO documented all the nursing interventions that they delivered to patients using the 10-item checklist (Refer to Table 1). In 2006 there were 10 PNOs and by the third year, there were 12 PNOs documenting their care in the system.

After the three-year period, for purposes of describing the type and frequency of interventions, we examined the nursing interventions within the 10 categories and then grouped the interventions according to the MSSS's (2008) four broad categories of PNO role functions (Refer to Table 2).

\section{Findings}

The types and frequency of interventions revealed through the analysis offered interesting information on the patterns of PNO interventions. Over the three years, the 12 PNOs documented a total of 43,906 nursing interventions (Refer to Table 3). Whether considered in total or by annual average according to the 10-category list, the majority of PNO specific interventions included those that

Table 3. Frequency of PNO interventions by year

\begin{tabular}{|c|c|c|c|c|c|c|c|c|}
\hline \multirow{2}{*}{ Intervention description } & \multicolumn{2}{|c|}{ 2006-2007 } & \multicolumn{2}{|c|}{ 2007-2008 } & \multicolumn{2}{|c|}{ 2008-2009 } & \multicolumn{2}{|c|}{ Average } \\
\hline & No. & $\%$ & No. & $\%$ & No. & $\%$ & No. & $\%$ \\
\hline Continuity of care & 1502 & 23.7 & 3933 & 22.8 & 3929 & 19.4 & 3121 & 21.9 \\
\hline Support to family \& patient & 883 & 13.9 & 3579 & 20.7 & 3972 & 19.6 & 2811 & 18.1 \\
\hline Monitor-surveillance & 859 & 13.6 & 2486 & 14.4 & 3526 & 17.4 & 2290 & 15.1 \\
\hline Teaching management of symptoms & 752 & 11.9 & 1759 & 10.2 & 1953 & 9.6 & 1488 & 10.6 \\
\hline Symptom assessment & 865 & 13.7 & 1615 & 9.3 & 1490 & 7.4 & 1323 & 10.1 \\
\hline Admin. or clerical & 547 & 8.6 & 1587 & 9.2 & 1931 & 9.5 & 1355 & 9.1 \\
\hline Referral-consult & 359 & 5.7 & 733 & 4.2 & 919 & 4.5 & 670 & 4.8 \\
\hline Data gathering & 278 & 4.4 & 716 & 4.1 & 1139 & 5.6 & 711 & 4.7 \\
\hline Advocacy & 158 & 2.5 & 507 & 2.9 & 1001 & 4.9 & 555 & 3.5 \\
\hline Family assessment & 136 & 2.2 & 369 & 2.1 & 423 & 2.1 & 309 & 2.1 \\
\hline Total & \multicolumn{2}{|c|}{6,339} & \multicolumn{2}{|c|}{17,284} & \multicolumn{2}{|c|}{20,283} & \multicolumn{2}{|c|}{14,635} \\
\hline
\end{tabular}


related to promoting coordination and continuity of care $(19.4 \%$ of interventions in year three), and those that involved providing support to patients and their families (19.6\% in year three). The least frequently used interventions involved family assessment $(2.1 \%$ in year three), and advocacy (4.9\% in year three).

When data were organized according to the PNO role functions (as per MSSS 2008), as described in Table 2, by the third year coordination of care comprised $38.4 \%$ of interventions, while assessment comprised $32.4 \%$, support $19.6 \%$ and teaching/information $9.6 \%$ (Refer to Figure 1). The pattern of functions was remarkably stable over the first three years of the PNO role implementation. After year one, the main change was an increase in support to patients and families.

\section{Discussion}

This is the first known prospective description reported in the literature of the types, variation, and frequency of interventions provided to patients and families by the PNO. These findings provide a longitudinal display of documented care and a broad overview of the interventions provided by the PNO. A consistent pattern was demonstrated over the three years, and the majority of the PNO interventions for patients with cancer and their families involved activities requiring nursing knowledge, clinical judgment and decision-making. The intervention categories provide a feasible approach to describe the practice patterns of the PNO. In addition, the interventions described within the four role functions are consistent with the major dimensions recommended by Fillion (2009) in the Professional Navigation Framework.

The patterns in this report have several implications for nursing practice, education, and research. The description of interventions of the PNO delivery model in this project is clearly within the domains of practice by a specialized oncology nurse. This description provides information about the distribution and dominance of interventions that are consistent with specific CANO domains of practice that may vary in distribution for specialized oncology nurses working in other settings (ambulatory clinics, hemato-oncology units, etc.)

The findings demonstrated that the predominant category of interventions provided by the PNO for a group of patients, nearing $40 \%$, is for coordination/continuity of care, and this addresses the CANO/ACIO domain to facilitate continuity of care/navigate the system. The next CANO/ACIO domain dominating PNO practice, comprising about a third of the interventions, is comprehensive health assessment, namely in this project the assessment of needs and symptoms. The CANO/ACIO domain supportive and therapeutic relationships represents $20 \%$ of the PNO interventions described as support. The description of the dominant CANO/ACIO practice domains in the PNO role highlights the uniqueness of this specialized oncology nurse. Other specialized oncology nurses are also practising according to the domains of practice, but with a different emphasis and dose intensity that could be explained by the differences in model of care delivery (e.g., oncology day centre, in-patient units, etc.).

The PNO is a health care professional who is dedicated to providing patients with cancer and their families with constant and consistent supportive care throughout the entire care trajectory. The PNO is a member of an interdisciplinary team, and works in collaboration and partnership with patients, families, and other health professionals. The delivery model approach is population-based (i.e., cancer type or program) rather than services-related (i.e., in-patient or out-patient) and provides patients and their families with a reliable resource person over time and on a consistent basis. The PNO has the advantage, in contrast to the nurses working in a hematooncology unit, of being flexible with her time and caseload organization and can act autonomously to schedule patient encounters and to meet with different team members, departments, and com- munity resources. The benefit to patients is that the PNO can consistently facilitate continuity of care across the usual boundaries of service delivery.

More than $90 \%$ of the interventions delivered by the PNO involved activities that require the use of: the nursing process, clinical oncology expertise, and specialized oncology nursing competencies/ knowledge. This information is helpful to tailor the orientation of nurses assuming the role, as well as to guide administrators and educators in the provision of appropriate continuing education activities to support the PNO. The expertise needed to assess complex symptoms presented by patients with cancer, either over the telephone or during a nursing visit, requires a high level of knowledge and skills by the PNO. She must take into account the different aspects of the therapeutic plan that was proposed to the patient by the interdisciplinary team and the interventions of the other professionals involved in the situation, as well as new information the patient is presenting to fully assess the situation and problem-solve with the patient. She also needs communication and interpersonal skills including telephone interventions to reassure the patient, as well as work collaboratively with other team members.

Complex decisions by the PNO can relate, for example, to an acute medical situation that a patient presents with and for which the PNO needs to make a decision about the type and urgency of referral (e.g., radiation oncology, medical oncology, and/or psychology) and whether to book an early clinic appointment or to instruct a patient to seek immediate attention. This type of decision-making process requires nursing knowledge and clinical judgment. The PNO is able to ensure that patients' needs are addressed and streamlined in an effective and efficient manner, and this ensures continuity of care. From an administrative perspective, filling such a role with a nurse is beneficial for resource allocation because this one individual is able to manage patients' clinical and organizational needs. Our experience and the profile of interventions described in this paper led us to conclude that patient needs are best addressed through the use of a PNO.

The intervention classification scheme is appropriate not only to describe the practice patterns of the PNO. It could also be used as a basis for developing a measure of the intensity of interventions (of the clinical activities) for patients and their families. This project provides the foundation for further exploration of the impact of the specific interventions on patient outcomes. It is important to further our understanding of the interventions that PNOs provide and their impact on outcomes in order to improve patient care.

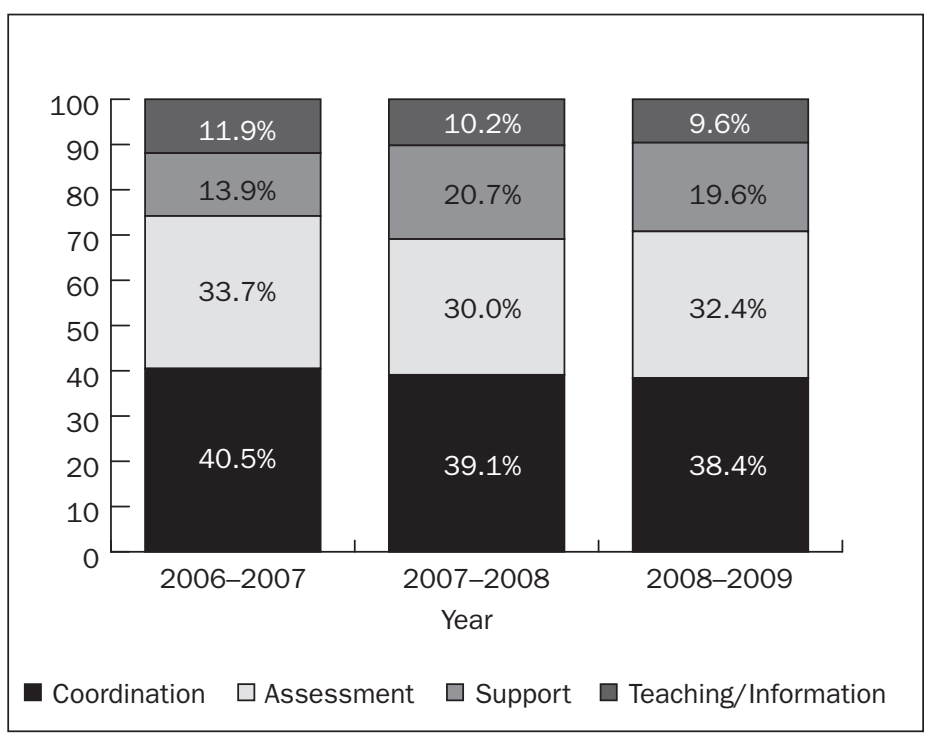

Figure 1. Distribution (\%) of role functions by year 


\section{Limitations}

There are limitations to take into consideration when reviewing the intervention patterns reported here. First, the data were gathered using self-reported activities by nurses. We do not have validated data that could come from observation of practice. Second, we have not examined differences between interventions involving either in-person visits or telephone calls. Third, interventions reported here are a summary of patients with a variety of cancers; patterns specific to cancer type were not examined. The patterns of interventions may vary with cancer type or stage.

\section{Conclusions}

We have provided a broad overview of interventions documented by the PNO. The majority of interventions encompassed activities that require nursing clinical knowledge, judgment and decision-making. The actual description of the PNO interventions presented in this report validated the Quebec model and demonstrated the feasibility and pertinence of the implementation of this new nursing role. Our analysis defines and describes the prevalence of the interventions

\section{References}

Allard, N.C. (2007). Day surgery for breast cancer: Effects of a psychoeducational telephone intervention on functional status and emotional distress. Oncology Nursing Forum, 34, 133-141.

Canadian Partnership Against Cancer. (2010, January). Cancer Journey: Guide to Implementing Navigation [Working copy]. Retrieved from www.partnershipagainstcancer.ca/sites/default/ files/Guide_Implementation_Navigation.pdf

de Serres, M., \& Beauchesne, N. (2000). L'intervenant pivot en oncologie: un rôle d'évaluation, d'information et de soutien pour le mieux-être des personnes atteintes de cancer. Québec: Conseil québécois de lutte contre le cancer.

Dumont, I., Dumont, S., \& Turgeon, J. (2005). Continuity of care for advanced cancer patients. Journal of Palliative Care, 21, 49-56.

Ferrante, J.M., Chen, P.H., \& Kim, S. (2008). The effect of patient navigation on time to diagnosis, anxiety, and satisfaction in urban minority women with abnormal mammograms: A randomized controlled trial. Journal of Urban Health, 85, 114-124.

Fillion, L., de Serres, M., Lapointe-Goupil, R., Bairati, I., Gagnon, P., Deschamps, M. et al. (2006). Implementing the role of patientnavigator nurse at a university hospital centre. Canadian Oncology Nursing Journal, 16(1), 11-17.

Fillion, L., de Serres, M., Cook, S., Goupil, R. L., Bairati, I., \& Doll, R. (2009). Professional patient navigation in head and neck cancer. Seminars Oncology Nursing, 25, 212-221.

Fischer, S.M., Sauaia, A., \& Kutner, J.S. (2007). Patient navigation: A culturally competent strategy to address disparities in palliative care. Journal of Palliative Medicine, 10, 1023-1028.

Fitch, M., Porter, H.B., \& Page, B.D. (2009). Supportive Care Framework: A Foundation for Person-Centred Care. Pembroke, ON: Pappin Communications.

Freeman, H.P., Muth, B.J., \& Kerner, J.F. (1995). Expanding access to cancer screening and clinical follow-up among the medically underserved. Cancer Practice, 3, 19-30.

Haggerty , J.L., Reid, R.J. Freeman, G.K., Starfield, B.H., Adair, C.E., \& McKendr, R. (2003). Continuity of care: A multidisciplinary review. BMJ. 32, 1219-1221.

Honea, N.J., Brintnall, R., Given, B., Sherwood, P., Colao, D.B., Somers, S.C., et al. (2008). Putting evidence into practice: Nursing assessment and interventions to reduce family caregiver strain and burden. Clinical Journal of Oncology Nursing, 12, 507-516.

Lohfeld, L., Brazil, K., \& Willison, K. (2007). Front line dispatch. Continuity of care for advanced cancer patients: Comparing the views of spousal caregivers in Ontario, Canada, to Dumont et al.'s theoretical model. Journal of Palliative Care, 23, 117-126. related to the four practice functions. The findings show that coordination/continuity of care and assessment of needs and symptoms are predominant role functions of the PNO and highlight the PNO role as a professional navigator. The findings provide sufficient clarity around the specific type of interventions and knowledge needed to implement them to suggest that this is a role for a specialized oncology nurse. Research is needed to examine how the interventions correspond to the needs of patients diagnosed with cancer. The patterns reported here provide the foundation to begin further empirical study of this important specialized nursing role. Through furthering our understanding of the process of care by the PNO and its impact on patient and system outcomes, we can build toward improving the quality of care for patients with cancer and their families.

\section{Acknowledgements}

To the pivot nurses in oncology at the CUSM who entered their data rigorously and continuously for more than three years.

Marika Swidzinski, RN, MEd, CON(C), Nurse Manager,

Hematology-Oncology, MUHC.

Martin, K. (2004). The Omaha System: A key to practice, documentation, and information management (2nd ed.). St. Louis, MS: Elsevier Saunders.

Miaskowski, C., Shockney, L., \& Chlebowski, R.T. (2008). Adherence to oral endocrine therapy for breast cancer: A nursing perspective. Clinical Journal of Oncology Nursing, 12, 213-221.

Ministère de la Santé et des Services sociaux (MSSS). (2005). Programme québécois de lutte contre le cancer. Pour optimiser la contribution des infirmières à la lutte contre le cancer. Comité de l'évolution de la pratique infirmière en oncologie (CEPIO). Retrieved from http://www.msss.gouv.qc.ca/sujets/prob_sante/ cancer/index.php?aid=30

Ministère de la Santé et des Services sociaux (MSSS) (2008). Programme québécois de lutte contre le cancer. Rôle de l'infirmière pivot en oncologie. Comité consultatif des infirmières en oncologie. Retrieved from http://www.msss.gouv.qc.ca/ sujets/prob_sante/cancer/index.php?aid=30

Mitchell, S.A., Beck, S.L., Hood, L.E., Moore, K., \& Tanner, E.R. (2007). Putting evidence into practice: Evidence-based interventions for fatigue during and following cancer and its treatment. Clinical Journal of Oncology Nursing, 11, 99.

Morgan, M.A. (2009). Considering the patient-partner relationship in cancer care: Coping strategies for couples. Clinical Journal of Oncology Nursing, 13, 65-72.

Plante, A., \& Joannette, S. (2009). L'intégration des infirmières pivots dans les équipes d'oncologie en Montérégie: un aspect de l'implémentation du Programme de lutte contre le cancer, Partie 1. Canadian Oncology Nursing Journal, 19(1), 13-18.

Rahm, A.K., Sukhanova, A., Ellis, J., \& Mouchawar, J. (2007). Increasing utilization of cancer genetic counseling services using a patient navigator model. Journal of Genetic Counseling, 16, 171-177.

Saegrov, S., \& Halding, A. (2004). What is it like living with the diagnosis of cancer? European Journal of Cancer Care, 13, 145153.

Schwaderer, K.A., \& Itano, J.K. (2007). Bridging the healthcare divide with patient navigation: Development of a research program to address disparities. Clinical Journal of Oncology Nursing, 11, 633-639.

Skrutkowski, M., Saucier, A., Eades, M., Swidzinski, M., Ritchie, J., \& Marchionni, C. (2008). Impact of a pivot nurse in oncology on patients with lung or breast cancer: Symptom distress, fatigue, quality of life, and use of healthcare resources. Oncology Nursing Forum, 35, 948-954. 